\title{
Polaron formation in the vicinity of a narrow Feshbach resonance
}

\author{
W. Casteels and M. Wouters \\ TQC, Universiteit Antwerpen, Universiteitsplein 1, 2610 Wilrijk, Belgium
}

(Received 3 July 2014; published 3 October 2014)

\begin{abstract}
The polaronic system consisting of an impurity in a dilute Bose-Einstein condensate is considered in the presence of a narrow Feshbach resonance. For this purpose a coupled-channel model is used, which at the mean-field level predicts the formation of quasiparticles that are a superposition of the impurity and the molecular states. The impurity-boson interactions and the coupling between the open and closed channels are then considered weak and a perturbative calculation of the corrections to the mean-field results is presented. This allows us to examine the properties of the quasiparticles, such as the lifetime and the effective mass. The relation between the model parameters and the experimental parameters is determined by identifying the low-energy $T$ matrix and applying a proper renormalization scheme.
\end{abstract}

DOI: 10.1103/PhysRevA.90.043602

PACS number(s): 03.75.Hh, 67.85.Bc, 71.36.+c, 71.38.Fp

\section{INTRODUCTION}

In recent years ultracold atomic systems have become increasingly popular as quantum simulators for many-particle models [1]. These systems are attractive since they are extremely clean as compared to a typical solid-state experiment and because of the extreme controllability and tunability. It is, for example, possible to experimentally vary the interatomic interactions by means of a Feshbach resonance. This is a scattering resonance which occurs when the scattering energy approaches the binding energy of a molecular state (see, for example, Ref. [2] for a review on Feshbach resonances in ultracold gases). If the molecule and the scattering atoms have a different magnetic moment, the difference in energy can be varied with an external magnetic field. This allows us to experimentally tune the interatomic interactions as a function of an external magnetic field. A distinction is typically made between a broad or entrance channel dominated resonance and a narrow or closed channel dominated resonance [2]. A broad resonance can be well described by a single-channel model with an effective interaction amplitude, while for the description of a narrow resonance the molecular state has to be described explicitly with a coupled-channel model [3].

Recently the principle of quantum simulation with ultracold gases has been considered for the Fröhlich polaron model [4-6]. The polaron is well known from solid-state physics, where it denotes a charge carrier in a charged lattice. If the Bogoliubov approximation is valid the Hamiltonian of an impurity in a Bose-Einstein condensate can be mapped onto the Fröhlich polaron Hamiltonian [4-6]. This setup is particularly attractive since the polaronic coupling parameter depends on the interatomic interaction strength, which is tunable with a Feshbach resonance, whereas in the solid-state context this is a material constant. An example of a promising experiment to probe the polaronic strong-coupling regime is the doping of an ultracold $\mathrm{Rb}$ gas with single $\mathrm{Cs}$ impurity atoms from Ref. [7] since it was shown that this system exhibits a broad interspecies Feshbach resonance [8]. On the other hand there are also binary systems that exhibit only narrow interspecies Feshbach resonances, as, for example, a ${ }^{6} \mathrm{Li}-\mathrm{Na}$ mixture [9], in which case the exact influence of the resonance on the polaronic properties is not clear.
In this paper we are particularly interested in the polaronic system in the presence of a narrow Feshbach resonance. A narrow Feshbach resonance corresponds to weak coupling to the molecular channel so that perturbation theory is applicable to describe the corrections to the mean-field results. The experimental relevance of this regime is demonstrated by the wide range of resonance widths that have been observed with ultracold gases, including narrow resonances [2].

Recently the presence of a Feshbach resonance has also been predicted and observed in the context of polaritons [10-12]. This system is reminiscent of the polaronic system considered in this paper and can in principle be described by the same model Hamiltonian. However, due to the inevitable losses these systems are not at equilibrium, while in this paper the system is considered at equilibrium and the results are thus not expected to be applicable for the current experiments. If on the other hand the loss rate could be made sufficiently low such that the system is in a quasithermal equilibrium the presented derivation is expected to be valid.

The structure of this manuscript is as follows. First the model Hamiltonian describing two types (denoted as spin up and spin down) of particles that can form a molecule is introduced. The manifestation of a Feshbach resonance within this model is investigated by calculating the two-body $T$ matrix, and a renormalization scheme is discussed. Then, the spin-up particles are assumed to form a dilute Bose-Einstein condensate by applying the Bogoliubov approximation. The mean-field part of the Hamiltonian is then diagonalized with another Bogoliubov transformation resulting in two types of quasiparticles consisting of a superposition of the spin-down particles and the molecules. The interactions are then assumed to be weak and perturbation theory is used to calculate the lowest-order corrections to the self-energies of the quasiparticles. This model is then applied for an impurity atom in a Bose-condensed atomic gas in three dimensions and the results are numerically studied for a ${ }^{6} \mathrm{Li}$ impurity in a $\mathrm{Na}$ condensate. Finally, the conclusions are presented.

\section{THE MODEL SYSTEM}

The following Hamiltonian is considered for the model system (for a derivation from the atomic Hamiltonian see, for 
example, Refs. [13-15]):

$$
\begin{aligned}
\widehat{H}= & \sum_{\vec{k}} \sum_{\sigma=\uparrow, \downarrow} \xi_{\vec{k}, \sigma} \widehat{a}_{\vec{k}, \sigma}^{\dagger} \widehat{a}_{\vec{k}, \sigma}+\sum_{\vec{k}} \varepsilon_{\vec{k}}^{M} \widehat{b}_{\vec{k}}^{\dagger} \widehat{b}_{\vec{k}} \\
& +g \sum_{\vec{k}, \vec{q}}\left(\widehat{b}_{\vec{k}}^{\dagger} \widehat{a}_{\vec{k}}+\vec{q}, \uparrow \widehat{a}_{\frac{\vec{k}}{2}-\vec{q}, \downarrow}+\text { H.c. }\right) \\
& +\frac{U^{\uparrow \uparrow}}{2} \sum_{\vec{k}, \vec{k}^{\prime}, \vec{q}} \widehat{a}_{\vec{k}+\vec{q}, \uparrow}^{\dagger} \widehat{a}_{\vec{k}^{\prime}-\vec{q}, \uparrow}^{\dagger} \widehat{a}_{\vec{k}^{\prime}, \uparrow} \widehat{a}_{\vec{k}, \uparrow} \\
& +U^{\uparrow \downarrow} \sum_{\vec{k}, \vec{k}^{\prime}, \vec{q}} \widehat{a}_{\vec{k}+\vec{q}, \uparrow}^{\dagger} \widehat{a}_{\vec{k}^{\prime}-\vec{q}, \downarrow}^{\dagger} \widehat{a}_{\vec{k}^{\prime}, \downarrow} \widehat{a}_{\vec{k}, \uparrow} .
\end{aligned}
$$

The first term represents the kinetic energy of the spin-up $(\sigma=\uparrow)$ and spin-down $(\sigma=\downarrow)$ particles with $\widehat{a}_{\vec{k}, \sigma}^{\dagger}\left(\widehat{a}_{\vec{k}, \sigma}\right)$ the creation (annihilation) operators for a particle with wave vector $\vec{k}$, spin $\sigma$, and $\xi_{\vec{k}, \sigma}=E_{\vec{k}, \sigma}-\mu_{\sigma}$, where $\mu_{\sigma}$ is the chemical potential and $E_{\vec{k}, \sigma}=E_{0, \sigma}+\hbar^{2} k^{2} /\left(2 m_{\sigma}\right)$ is the energy, with $m_{\sigma}$ the mass. The second term gives the energy of the free molecules, consisting of a bound state of a spin-up and a spin-down particle, with $\widehat{b}_{\vec{k}}^{\dagger}\left(\widehat{b}_{\vec{k}}\right)$ the creation (annihilation) operators for a molecule with wave vector $\vec{k}$ and $\varepsilon_{\vec{k}}^{M}=-\varepsilon^{M}+$ $\hbar^{2} k^{2} /(2 M)-\mu_{\uparrow}-\mu_{\downarrow}$, with $\varepsilon^{M}$ the binding energy and $M$ the mass. The first term on the second line represents the formation and dissociation of the molecule with interaction amplitude $g$. The next term is the interaction energy of the spin-up particles interacting with a contact potential with amplitude $U^{\uparrow \uparrow}$, and the last term represents the interaction between the spin-up and spin-down particles for which also a contact potential is considered, with amplitude $U^{\uparrow \downarrow}$. Note that no interactions between the spin-down particles are considered.

\section{A. Two-body $\boldsymbol{T}$ matrix and renormalization}

Only considering one spin-up and one spin-down particle allows a calculation of the two-body $T$ matrix of the model system with a derivation along the lines of Ref. [14]. This results in the following expression:

$$
\frac{1}{T^{\uparrow \downarrow}(E)}=\left(U^{\uparrow \downarrow}+\frac{|g|^{2}}{E-\varepsilon^{M}}\right)^{-1}-\sum_{\vec{q}} \frac{1}{E-\frac{\hbar^{2} q^{2}}{2 m_{r}}+i \delta},
$$

with $E$ the scattering energy, $\delta$ a positive infinitesimal, and $m_{r}$ the reduced mass: $m_{r}^{-1}=m_{\downarrow}^{-1}+m_{\uparrow}^{-1}$. Note that if the dimension is larger than 1 this expression contains a UV divergence which has to be renormalized, as shown in Ref. [14]. This results in the following renormalization scheme to express the two-body model parameters $U^{\uparrow \downarrow}, g$, and $\varepsilon^{M}$ in terms of the corresponding physical parameters $U_{P}^{\uparrow \downarrow}, g_{P}$, and $\varepsilon_{P}^{M}$ :

$$
\begin{aligned}
U^{\uparrow \downarrow} & =\frac{U_{P}^{\uparrow \downarrow}}{1+R U_{P}^{\uparrow \downarrow}}, \\
g & =\frac{g_{P}}{1+R U_{P}^{\uparrow \downarrow}}, \\
\varepsilon^{M} & =\varepsilon_{P}^{M}+R g g_{P},
\end{aligned}
$$

with

$$
R=\sum_{\vec{q}} \frac{1}{E-\frac{\hbar^{2} q^{2}}{2 m_{r}}} .
$$

Expressing the $T$ matrix (2) as a function of the physical parameters results in an expression that does not contain a UV divergence:

$$
T^{\uparrow \downarrow}(E)^{-1}=\left(U_{P}^{\uparrow \downarrow}+\frac{\left|g_{P}\right|^{2}}{E-\varepsilon_{P}^{M}}\right)^{-1}+i \pi g(E),
$$

where $g(E)$ is the density of states for a free gas with mass $m_{r}$. Note that the $\left|g_{P}\right|^{2} /\left(E-\varepsilon_{P}^{M}\right)$ term in (5) describes the Feshbach resonance.

\section{B. Coupled-channel versus single-channel model}

As mentioned in the Introduction a distinction is typically made between broad or entrance channel dominated and narrow or closed channel dominated resonances for Feshbach resonances [2]. As the name suggests the molecular channel is important for the closed channel dominated resonances and they should be described with a coupled-channel model, as, for example, described by Hamiltonian (1). For a narrow or entrance channel dominated resonance the occupation of the closed molecular channel is typically small and a single-channel model can be used with an effective interaction amplitude $U_{\text {eff }}^{\uparrow \downarrow}$ for the description:

$$
U_{\text {eff }}^{\uparrow \downarrow}=U_{P}^{\uparrow \downarrow}-\frac{\left|g_{P}\right|^{2}}{\varepsilon_{P}^{M}} .
$$

In the following a coupled-channel calculation is developed and analyzed in perturbation theory in $g_{P}$. Its results will be compared to a perturbative single-channel calculation.

\section{BOGOLIUBOV APPROXIMATION}

The temperature is assumed to be sufficiently low such that a macroscopic number of spin-up particles occupy the one-particle ground state and thus form a Bose-Einstein condensate. If the gas is dilute, the Bogoliubov approximation can be used, which transforms the Hamiltonian (1) into [16]

$$
\widehat{H}=E^{\mathrm{GP}}+\widehat{H}^{\mathrm{MF}}+\widehat{H}_{I} \text {. }
$$

The first term is the well-known Gross-Pitaevskii energy of the condensate [16]. The second term is the mean-field Hamiltonian, which consists of all the quadratic contributions:

$$
\begin{aligned}
\widehat{H}^{\mathrm{MF}}= & \sum_{\vec{k}}\left(\begin{array}{ll}
\widehat{a}_{\vec{k}}^{\dagger} & \widehat{b}_{\vec{k}}^{\dagger}
\end{array}\right)\left(\begin{array}{cc}
\xi_{\vec{k}, \downarrow}+U^{\uparrow \downarrow} n_{0} & g \sqrt{n_{0}} \\
g \sqrt{n_{0}} & \varepsilon_{\vec{k}}^{M}
\end{array}\right)\left(\begin{array}{c}
\widehat{a}_{\vec{k}} \\
\widehat{b}_{\vec{k}}
\end{array}\right) \\
& +\sum_{\vec{k} \neq 0} \varepsilon_{\vec{k}}^{\mathrm{Bog}_{\widehat{\alpha}_{\vec{k}}}^{\dagger} \widehat{\alpha}_{\vec{k}},}
\end{aligned}
$$

where $\left\{\widehat{a}_{\vec{k}}\right\}$ denotes the annihilation operators for the spindown particles. The condensate density is denoted as $n_{0}$ and the operators $\left\{\widehat{\alpha}_{\vec{k}}^{\dagger}\right\}$ and $\left\{\widehat{\alpha}_{\vec{k}}\right\}$ create and annihilate the Bogoliubov excitations with the corresponding Bogoliubov dispersion:

$$
\varepsilon_{\vec{k}}^{\text {Bog }}=\sqrt{\left(E_{\vec{k}}^{\uparrow}+U^{\uparrow \uparrow} n_{0}\right)^{2}-\left(U^{\uparrow \uparrow} n_{0}\right)^{2}},
$$


where the notation $E_{\vec{k}}^{\sigma}=E_{\vec{k}, \sigma}-E_{0, \sigma}$ was introduced. The operators $\widehat{\alpha}_{\vec{k}}^{\dagger}$ and $\widehat{\alpha}_{\vec{k}}$ are related to the spin-up operators with the Bogoliubov transformation (for $\vec{k} \neq \overrightarrow{0}$ ):

$$
\begin{aligned}
& \widehat{\alpha}_{\vec{k}}^{\dagger}=u_{k} \widehat{a}_{\vec{k}, \uparrow}^{\dagger}+v_{k} \widehat{a}_{-\vec{k}, \uparrow}, \\
& \widehat{\alpha}_{\vec{k}}=u_{k} \widehat{a}_{\vec{k}, \uparrow}+v_{k} \widehat{a}_{-\vec{k}, \uparrow}^{\dagger} .
\end{aligned}
$$

The coefficients $u_{k}$ and $v_{k}$ are given by

$$
\begin{aligned}
& v_{k}^{2}=\frac{1}{2}\left(\frac{E_{\vec{k}}^{\uparrow}+U^{\uparrow \uparrow} n_{0}}{\varepsilon_{\vec{k}}}-1\right), \\
& u_{k}^{2}=\frac{1}{2}\left(\frac{E_{\vec{k}}^{\uparrow}+U^{\uparrow \uparrow} n_{0}}{\varepsilon_{\vec{k}}}+1\right) .
\end{aligned}
$$

For the chemical potential of the condensate the lowest-order approximation $\mu_{\uparrow}=E_{0, \uparrow}+U^{\uparrow \uparrow} n_{0}$ is used. Note that this induces a shift of the molecular energy $\varepsilon_{\vec{k}}^{M}$. As a typical length scale of the condensate the healing length is introduced, defined as $\xi=\hbar\left(m n_{0} U^{\uparrow \uparrow}\right)^{-1 / 2}$.

The interaction Hamiltonian $\widehat{H}_{I}$ is given by

$$
\begin{aligned}
\widehat{H}_{I}= & \sum_{\vec{k}, \vec{q} \neq 0}\left[\left(g \widehat{b}_{\vec{q}+\vec{k}}^{\dagger} \widehat{a}_{\vec{k}}+U^{\uparrow \downarrow} \sqrt{n_{0}} \widehat{a}_{\vec{k}+\vec{q}}^{\dagger} \widehat{a}_{\vec{k}}\right)\left(u_{q} \widehat{\alpha}_{\vec{q}}-v_{q} \widehat{\alpha}_{-\vec{q}}^{\dagger}\right)\right. \\
& + \text { H.c. }] .
\end{aligned}
$$

From now on only a single spin-down particle is considered, which gives for the corresponding chemical potential $\mu_{\downarrow}=E_{0, \downarrow}$.

\section{DIAGONALIZATION OF THE MEAN-FIELD PART}

The mean-field Hamiltonian (8) can be diagonalized by applying the following unitary transformation:

$$
\left(\begin{array}{c}
\widehat{a}_{\vec{k}}^{\dagger} \\
\widehat{b}_{\vec{k}}^{\dagger}
\end{array}\right)=\left(\begin{array}{cc}
\beta_{\vec{k}} & -\alpha_{\vec{k}} \\
\alpha_{\vec{k}} & \beta_{\vec{k}}
\end{array}\right)\left(\begin{array}{c}
\widehat{\Phi}_{\vec{k}}^{\dagger} \\
\widehat{\Psi}_{\vec{k}}^{\dagger}
\end{array}\right),
$$

with

$$
\begin{aligned}
& \beta_{\vec{k}}^{2}=\frac{1}{2}\left(1+\frac{E_{\vec{k}}^{\downarrow}+U^{\uparrow \downarrow} n_{\uparrow}-\varepsilon_{\vec{k}}^{M}}{\sqrt{\left(E_{\vec{k}}^{\downarrow}+U^{\uparrow \downarrow} n_{0}-\varepsilon_{\vec{k}}^{M}\right)^{2}+4 g^{2} n_{0}}}\right), \\
& \alpha_{\vec{k}}^{2}=\frac{1}{2}\left(1-\frac{E_{\vec{k}}^{\downarrow}+U^{\uparrow \downarrow} n_{\uparrow}-\varepsilon_{\vec{k}}^{M}}{\sqrt{\left(E_{\vec{k}}^{\downarrow}+U^{\uparrow \downarrow} n_{0}-\varepsilon_{\vec{k}}^{M}\right)^{2}+4 g^{2} n_{0}}}\right) .
\end{aligned}
$$

The mean-field Hamiltonian (8) is then transformed into

$$
\widehat{H}^{\mathrm{MF}}=\sum_{\vec{k}}\left(\varepsilon_{\vec{k}}^{+} \widehat{\Phi}_{\vec{k}}^{\dagger} \widehat{\Phi}_{\vec{k}}+\varepsilon_{\vec{k}}^{-} \widehat{\Psi}_{\vec{k}}^{\dagger} \widehat{\Psi}_{\vec{k}}+\varepsilon_{\vec{k}}^{\mathrm{Bog}} \widehat{\alpha}_{\vec{k}}^{\dagger} \widehat{\alpha}_{\vec{k}}\right) .
$$

This shows the emergence of two quasiparticles that consist of a superposition of the impurity and the molecular state, with dispersions:

$$
\begin{aligned}
\varepsilon_{\vec{k}}^{ \pm}= & \frac{1}{2}\left[E_{\vec{k}}^{\downarrow}+U^{\uparrow \downarrow} n_{0}+\varepsilon_{\vec{k}}^{M}\right. \\
& \left. \pm \sqrt{\left(E_{\vec{k}}^{\downarrow}+U^{\uparrow \downarrow} n_{0}-\varepsilon_{\vec{k}}^{M}\right)^{2}+4 g^{2} n_{0}}\right] .
\end{aligned}
$$

The mean-field effective masses $m^{\mathrm{MF}( \pm)}$ can be determined from the behavior of the dispersion at small $k=|\vec{k}|$ :

$$
\frac{1}{m^{\mathrm{MF}( \pm)}}=\left.\frac{1}{\hbar^{2}} \frac{\partial^{2} \varepsilon_{\vec{k}}^{ \pm}}{\partial k^{2}}\right|_{k \rightarrow 0} .
$$

Applying the unitary transformation (14) for the interaction Hamiltonian (13) results in

$$
\begin{aligned}
\widehat{H}_{I}= & \sum_{\vec{q} \neq 0, \vec{k}}\left(\gamma_{\vec{k}+\vec{q}} \widehat{\Phi}_{\vec{k}+\vec{q}}^{\dagger}+\sigma_{\vec{k}+\vec{q}} \widehat{\Psi}_{\vec{k}+\vec{q}}^{\dagger}\right)\left(\beta_{k} \widehat{\Phi}_{\vec{k}}-\alpha_{k} \widehat{\Psi}_{\vec{k}}\right) \\
& \times\left(u_{q} \widehat{\alpha}_{\vec{q}}-v_{q} \widehat{\alpha}_{-\vec{q}}^{\dagger}\right)+\text { H.c. },
\end{aligned}
$$

where the following functions were introduced:

$$
\begin{aligned}
& \gamma_{\vec{k}}=g \alpha_{\vec{k}}+U^{\uparrow \downarrow} \sqrt{n_{0}} \beta_{\vec{k}}, \\
& \sigma_{\vec{k}}=g \beta_{\vec{k}}-U^{\uparrow \downarrow} \sqrt{n_{0}} \alpha_{\vec{k}} .
\end{aligned}
$$

\section{PERTURBATIVE CALCULATION OF THE SELF-ENERGY}

In general the Green's function of a particle with creation (annihilation) operators $\left\{\widehat{\Psi}_{\vec{k}}^{\dagger}\right\}\left(\left\{\widehat{\Psi}_{\vec{k}}\right\}\right)$ is defined as [17]

$$
G\left(\vec{k}, t-t^{\prime}\right)=-i\left\langle\mathcal{T} \widehat{\Psi}_{\vec{k}}(t) \widehat{\Psi}_{\vec{k}}^{\dagger}\left(t^{\prime}\right)\right\rangle,
$$

where $\mathcal{T}$ is the time-ordering operator. The frequency representation of the Green's function is given by the Fourier transform with respect to the time

$$
G(\vec{k}, \omega)=\int_{-\infty}^{\infty} d t e^{i \omega t} G(\vec{k}, t)
$$

and can typically be written as a function of the self-energy $\Sigma(\vec{k}, \omega)$ :

$$
G(\vec{k}, \omega)=\frac{\hbar}{\hbar \omega-\varepsilon_{\vec{k}}-\hbar \Sigma(\vec{k}, \omega)} .
$$

In the Appendix some details are provided on the perturbative calculation of the lowest nonvanishing contribution of the interaction Hamiltonian (13) to the self-energy $\Sigma(\vec{k}, \omega)$. The results for the two branches are presented in Fig. 1 by

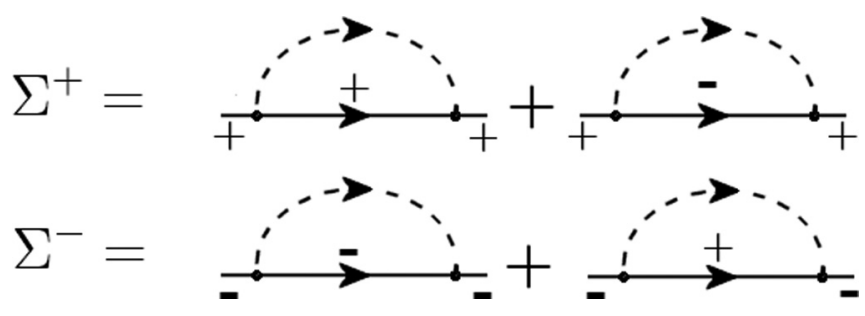

FIG. 1. The Feynman diagrams corresponding to the expressions (25) for the self-energies of the two branches. The dashed lines denote a Bogoliubov excitation and the full lines are the unperturbed upper $(+)$ and lower $(-)$ quasiparticles. 
Feynman diagrams and given by

$$
\begin{aligned}
\Sigma^{-}(\vec{k}, \omega)= & \frac{1}{\hbar^{2}} \sum_{\vec{q}}\left[\left(u_{\vec{q}} \sigma_{\vec{k}} \alpha_{\vec{k}-\vec{q}}-v_{\vec{q}} \alpha_{\vec{k}} \sigma_{\vec{k}-\vec{q}}\right)^{2} G_{0}^{-}\left(\vec{k}-\vec{q}, \omega-\omega_{\vec{q}}\right)\right. \\
& \left.+\left(u_{\vec{q}} \sigma_{\vec{k}} \beta_{\vec{k}-\vec{q}}+v_{\vec{q}} \gamma_{\vec{k}-\vec{q}} \alpha_{\vec{k}}\right)^{2} G_{0}^{+}\left(\vec{k}-\vec{q}, \omega-\omega_{\vec{q}}\right)\right], \\
\Sigma^{+}(\vec{K}, \omega)= & \frac{1}{\hbar^{2}} \sum_{\vec{q}}\left[\left(v_{\vec{q}} \beta_{\vec{k}} \gamma_{\vec{k}-\vec{q}}-u_{\vec{q}} \gamma_{\vec{k}} \beta_{\vec{k}-\vec{q}}\right)^{2} G_{0}^{+}\left(\vec{k}-\vec{q}, \omega-\omega_{\vec{q}}\right)\right. \\
& \left.+\left(u_{\vec{q}} \gamma_{\vec{k}} \alpha_{\vec{k}-\vec{q}}+v_{\vec{q}} \sigma_{\vec{k}-\vec{q}} \beta_{\vec{k}}\right)^{2} G_{0}^{-}\left(\vec{k}-\vec{q}, \omega-\omega_{\vec{q}}\right)\right] .
\end{aligned}
$$

These expressions are valid if the following two conditions are satisfied (with $D$ the dimension):

$$
\begin{aligned}
\frac{1}{n_{0} \xi^{D}}\left(\frac{U^{\uparrow \downarrow}}{U^{\uparrow \uparrow}}\right)^{2} & \ll 1, \\
\frac{1}{n_{0} \xi^{D}}\left(\frac{g}{\sqrt{n_{0}} U^{\uparrow \uparrow}}\right)^{2} & \ll 1 .
\end{aligned}
$$

Condition (26a) shows that the average distance between the particles should be small as compared to the healing length and the background interactions where condition (26b) requires that the coupling to the molecule should be weak as compared to the condensate interactions. The latter condition implies that our approximation is only valid for narrow Feshbach resonances (small $g$ ).

If there is no coupling with the molecule $(g=0)$ the expressions (25) reduce to the well-known weak-coupling result for the BEC-impurity Fröhlich polaron [18,19]:

$$
\Sigma^{\mathrm{pol}}(\vec{k}, \omega)=\frac{n_{0}\left(U^{\uparrow \downarrow}\right)^{2}}{\hbar} \sum_{\vec{q}} \frac{E_{\vec{q}}^{\uparrow}}{\varepsilon_{\vec{q}}^{\text {Bog }}} \frac{1}{\hbar \omega-\hbar \omega_{\vec{q}}-E_{\vec{k}-\vec{q}}^{\downarrow}+i \delta} .
$$

The physical states of the system correspond to the poles of the Green's function (24). Since the self-energy is assumed small with respect to the mean-field dispersion, the argument $\omega$ of the self-energy can be replaced by the unperturbed energy $\varepsilon_{\vec{k}}^{ \pm}$ for the determination of the poles of (24). This results in the following expression for the energies of the quasiparticles:

$$
E_{\vec{k}}^{ \pm}=\varepsilon_{\vec{k}}^{ \pm}+\hbar \operatorname{Re}\left[\Sigma^{ \pm}\left(\vec{k}, \varepsilon_{\vec{k}}^{ \pm}\right)\right] .
$$

The inverse of the imaginary part of the self-energy gives the lifetime of the quasiparticles $\tau_{\vec{k}}^{ \pm}$:

$$
\tau_{\vec{k}}^{ \pm}=\operatorname{Im}\left[\Sigma_{0}\left(\vec{k}, \varepsilon_{\vec{k}}^{ \pm}\right)\right]^{-1} .
$$

We are also interested in the effective masses $m^{ \pm}$, which can be determined as

$$
\begin{aligned}
\frac{1}{m^{ \pm}} & =\left.\frac{1}{\hbar^{2}} \frac{\partial^{2} E_{\vec{k}}^{ \pm}}{\partial k^{2}}\right|_{k=0} \\
& =\frac{1}{m^{\mathrm{MF}( \pm)}}+\frac{1}{m^{\text {pert( }( \pm)}}
\end{aligned}
$$

where $m^{\mathrm{MF}( \pm)}$ is the mean-field effective mass (18) and the second term is the perturbative correction. Since this correction is assumed to be small we can write

$$
m^{ \pm} \approx m^{\mathrm{MF}( \pm)}\left(1-\frac{m^{\mathrm{MF}( \pm)}}{m^{\operatorname{pert}( \pm)}}\right)
$$

\section{Renormalization}

A closer look at the arguments of the $\vec{q}$ summations in the expressions for the self-energies (25) reveals a UV divergency if the dimension is larger than 1. This can be cured by using the renormalization equations (3). Since only the lowest-order contribution of a perturbation series is considered, the renormalizing equations have to be considered to the same order. Inserting the renormalization equations (3) into the expressions for the mean-field energies (17) and only considering the lowest-order terms of an expansion in $U R$ and $g^{2} R$ results in

$$
\begin{aligned}
& \varepsilon_{\vec{k}}^{-} \rightarrow \varepsilon_{\vec{k}}^{-}+\frac{\sigma_{\vec{k}}^{2}}{\hbar^{2}} \sum_{\vec{q}} \frac{1}{\frac{\hbar^{2} q^{2}}{2 m_{r}}-E}, \\
& \varepsilon_{\vec{k}}^{+} \rightarrow \varepsilon_{\vec{k}}^{+}+\frac{\gamma_{\vec{k}}^{2}}{\hbar^{2}} \sum_{\vec{q}} \frac{1}{\frac{\hbar^{2} q^{2}}{2 m_{r}}-E} .
\end{aligned}
$$

Here, it is understood that the expressions on the right-hand side are in terms of the physical coupling parameters $U_{P}^{\uparrow \downarrow}, g_{P}$, and $\varepsilon_{P}^{M}$. Moreover, since the results for the self-energies (25) are already of the order we are interested in, the bare coupling parameters can be simply replaced by the physical ones in these expressions. Using (33a) for the first term in the expression for the energies (28) exactly cancels the UV divergence.

\section{PHYSICAL SYSTEM}

The relation between the experimental parameters and the model parameters is now derived. The two-body $s$-wave scattering amplitude $f_{k}$ for the scattering of a spin-up and a spin-down atom is related to the $T$ matrix (5) as

$$
f_{k}=-\frac{m_{r}}{2 \pi \hbar^{2}} T\left(\frac{\hbar^{2} k^{2}}{2 m_{r}}\right)
$$

The inverse scattering amplitude is typically expanded for small $k$ as

$$
-\frac{1}{f_{k}}=\frac{1}{a}+i k-\frac{1}{2} r_{e} k^{2}+O\left(k^{4}\right)
$$


where $a$ is the scattering length, which for the model is given by

$$
a=\frac{m_{r}}{2 \pi \hbar^{2}}\left(U_{P}^{\uparrow \downarrow}-\frac{\left|g_{P}\right|^{2}}{\varepsilon}\right) .
$$

The effective range $r_{e}$ is related to the Feshbach width $R^{*}$ as $R^{*}=-r_{e} / 2$, which for the model is

$$
R^{*}=R_{\mathrm{res}}^{*}\left(1-\frac{a_{\mathrm{BG}}}{a}\right)^{2},
$$

with $R_{\text {res }}^{*}$ the value at resonance:

$$
R_{\mathrm{res}}^{*}=\frac{\pi \hbar^{4}}{\left|g_{P}\right|^{2} m_{r}^{2} V} .
$$

Note that, since our approximation is only valid for small $g_{P}$, $R_{\text {res }}^{*}$ should be large. The background scattering length $a_{\mathrm{BG}}$ is

$$
a_{\mathrm{BG}}=\frac{m_{r}}{2 \pi \hbar^{2}} U_{P}^{\uparrow \downarrow} .
$$

Typically the scattering length in the vicinity of a magnetic Feshbach resonance is parametrized as

$$
a=a_{\mathrm{BG}}\left(1-\frac{\Delta B}{B-B_{0}}\right),
$$

with $B$ the magnetic field, $B_{0}$ the location of the resonance, and $\Delta B$ the width, which are experimental parameters. In order to identify the scattering length of the model (36) with the physical scattering length (40) in the vicinity of the resonance, an expansion of the molecular energy dependence on the magnetic field is performed:

$$
\varepsilon(B)=\delta \mu\left(B-B_{0}\right)+O\left(\left(B-B_{0}\right)^{2}\right),
$$

where $\delta \mu$ is the difference between the magnetic moment of the separated atoms and the magnetic moment of the molecular state. This identification shows that the model parameters can be expressed as a function of the experimental parameters as follows:

$$
\begin{aligned}
U_{P}^{\uparrow \downarrow} & =\frac{2 \pi \hbar^{2} a_{\mathrm{BG}}}{m_{r}}, \\
\left|g_{P}\right|^{2} & =U_{P}^{\uparrow \downarrow} \delta \mu \Delta B .
\end{aligned}
$$

Note that $\Delta B$ needs to have the same sign as the background scattering length.

For the Bose gas the amplitude of the contact interactions is given by

$$
U^{\uparrow \uparrow}=\frac{4 \pi \hbar^{2} a_{\mathrm{BB}}}{m_{\uparrow}},
$$

with $a_{\mathrm{BB}}$ the boson-boson scattering length. The two conditions (26) for the perturbative result to be valid can be written as a function of the experimental parameters:

$$
\begin{aligned}
& 2 \pi^{3 / 2} \sqrt{n_{0} a_{\mathrm{BB}}^{3}}\left(\frac{a_{\mathrm{BG}}}{a_{\mathrm{BB}}}\right)^{2}\left(\frac{m_{\uparrow}}{m_{r}}\right)^{2}=\pi \alpha\left(\frac{m_{\uparrow}}{m_{r}}\right)^{2} \ll 1, \\
& 4 \pi^{3 / 2} \sqrt{n_{0} a_{\mathrm{BB}}^{3}} \frac{m_{\uparrow}}{m_{r}} \frac{a_{\mathrm{BG}}}{a_{\mathrm{BB}}} \frac{\delta \mu \Delta}{n_{0} U_{\uparrow}} \ll 1,
\end{aligned}
$$

where the first condition was also expressed as a function of the dimensionless polaronic coupling parameter $\alpha=a_{\mathrm{BG}}^{2} /$ $\left(a_{\mathrm{BB}} \xi\right)$ [6]. This shows that the first condition (44a) expresses the diluteness condition $n_{0} a_{\mathrm{BB}}^{3} \ll 1$, weak background interactions, and that the masses should not be too different. The second condition expresses the same and additionally that the width of the resonance $\Delta B$ should be small, corresponding to a narrow Feshbach resonance.

Since ultralow temperatures are considered the limit $E \rightarrow 0$ can be taken in the renormalization expressions (3).

\section{RESULTS AND DISCUSSION}

In the following we start by summarizing a few analytical results for the background (vanishing coupling to the molecule) and show how the perturbative single-channel results are obtained. Then the results are examined for the specific system of a ${ }^{6} \mathrm{Li}$ impurity in a $\mathrm{Na}$ condensate with an interspecies narrow Feshbach resonance.

\section{A. Background results and perturbative single-channel model}

If there is no coupling to the molecule $(g=0)$ the real part of the impurity self-energy (27) at $k=0$ is given by

$$
\begin{aligned}
& \frac{\operatorname{Re}\left[\hbar \Sigma^{\mathrm{BG}}(0)\right]}{\hbar^{2} /\left(m_{\uparrow} \xi^{2}\right)} \\
& =\frac{1}{2 \pi^{2}} \frac{1}{n_{0} \xi^{3}}\left(\frac{a_{\mathrm{BG}}}{a_{\mathrm{BB}}}\right)^{2} \frac{1+\frac{m_{\uparrow}}{m_{\downarrow}}}{1-\frac{m_{\downarrow}}{m_{\uparrow}}} \\
& \quad \times\left(1+\frac{\left(\frac{m_{\downarrow}}{m_{\uparrow}}\right)^{2}}{\sqrt{1-\left(\frac{m_{\downarrow}}{m_{\uparrow}}\right)^{2}}} \ln \left[\frac{m_{\uparrow}-\sqrt{m_{\uparrow}^{2}-m_{\downarrow}^{2}}}{m_{\downarrow}}\right]\right) .
\end{aligned}
$$

The imaginary part of the self-energy is only nonzero if $k>k_{c}=m_{\downarrow} /\left(m_{\uparrow} \xi\right)$. This corresponds to the Landau criterion for superfluidity [20] which states that energy can only be dissipated to the condensate if $k>k_{c}$. In this case the imaginary part is given by

$$
\begin{aligned}
\frac{\operatorname{Im} \Sigma^{\mathrm{BG}}\left(k>k_{c}\right)}{\hbar /\left(m_{\uparrow} \xi^{2}\right)}= & -\frac{1}{16 \pi} \frac{1}{n_{0} \xi^{3}} \frac{1}{\xi k}\left(\frac{a_{\mathrm{BG}}}{a_{\mathrm{BB}}}\right)^{2} \frac{\left(m_{\downarrow}+m_{\uparrow}\right)^{2}}{m_{\downarrow} m_{\uparrow}} \\
& \times\left(\frac{Q_{c}}{2} \sqrt{4+Q_{c}^{2}}-2 \sinh ^{-1}\left[\frac{Q_{c}}{2}\right]\right),
\end{aligned}
$$

with

$$
Q_{c}=\frac{2 \frac{m_{\downarrow}}{m_{\uparrow}} \sqrt{1-\left(\frac{m_{\downarrow}}{m_{\uparrow}}\right)^{2}+\xi^{2} k^{2}}-2 \xi k}{\left(\frac{m_{\downarrow}}{m_{\uparrow}}\right)^{2}-1} .
$$

The effective mass $m_{\downarrow}^{*}$ is given by

$$
\begin{aligned}
\frac{m_{\downarrow}}{m_{\downarrow}^{*}}= & 1+\frac{1}{6 \pi^{2}} \frac{1}{n_{0} \xi^{3}}\left(\frac{a_{\mathrm{BG}}}{a_{\mathrm{BB}}}\right)^{2} \frac{1}{\left(1-\frac{m_{\downarrow}}{m_{\uparrow}}\right)^{2}} \\
& \times\left(3+\frac{2+\left(\frac{m_{\downarrow}}{m_{\uparrow}}\right)^{2}}{\sqrt{1-\left(\frac{m_{\downarrow}}{m_{\uparrow}}\right)^{2}}} \ln \left[\frac{m_{\uparrow}-\sqrt{m_{\uparrow}^{2}-m_{I}^{2}}}{m_{\downarrow}}\right]\right) .
\end{aligned}
$$




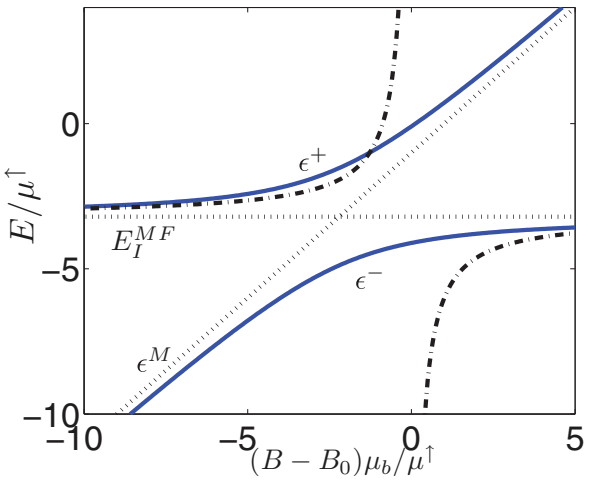

FIG. 2. (Color online) The quasiparticle dispersions $\varepsilon^{ \pm}$at the mean-field level [Eq. (17)] due to the mixing of the energy levels of the molecule and the ${ }^{6} \mathrm{Li}$ impurity in the presence of a Na condensate as a function of the magnetic field $B$ at $k=0$. The dotted lines are the energies of the impurity and the molecule which are shifted due to the presence of the condensate: $E_{I}^{\mathrm{MF}}=n_{0} U_{P}^{\uparrow \downarrow}$ and $\varepsilon^{M}=-\varepsilon_{0}^{M}-n_{0} U^{\uparrow \uparrow}$. The dash-dotted line gives the single-channel result.

The perturbative single-channel model corresponds to these background results with the replacement of the background scattering length $a_{\mathrm{BG}}$ with the Feshbach enhanced scattering length (40).

\section{B. Narrow Feshbach resonance}

The results are now studied for a ${ }^{6} \mathrm{Li}$ impurity in a $\mathrm{Na}$ condensate, corresponding to the parameters $m_{\downarrow} / m_{\uparrow}=0.263$ and $a_{\mathrm{BG}} / a_{\mathrm{BB}}=-1.336[9,21]$. In Ref. [9] various narrow interspecies Feshbach resonances are determined for this system. We will not focus on a specific resonance but examine the typical expected behavior. The difference in magnetic moment $\delta \mu$ is typically of the order of the Bohr magneton: $\delta \mu=e \hbar /\left(2 m_{e}\right)$, with $e$ the elementary charge and $m_{e}$ the electron mass. A coupled-channel calculation in Ref. [9] revealed that the widths of the resonances $\Delta B$ are of the order of $\mathrm{mG}$ so we take for the width $\Delta B=-1 \mathrm{mG}$. For the $\mathrm{Na}$ condensate the typical density $n_{0}=10^{14} \mathrm{~cm}^{-3}$ is considered. With these parameters we find

$$
\begin{array}{r}
2 \pi^{3 / 2}\left(n_{0} a_{\mathrm{BB}}^{3}\right)^{1 / 2}\left(\frac{a_{\mathrm{BG}}}{a_{\mathrm{BB}}}\right)^{2}\left(\frac{m_{\uparrow}}{m_{r}}\right)^{2} \approx 0.25, \\
4 \pi^{3 / 2}\left(n a_{\mathrm{BB}}^{3}\right)^{1 / 2} \frac{m_{\uparrow}}{m_{r}} \frac{a_{\mathrm{BG}}}{a_{\mathrm{BB}}} \frac{\delta \mu \Delta B}{n_{0} U^{\uparrow}} \approx 0.067 .
\end{array}
$$

This shows that the conditions (44) are satisfied and the system is in the perturbative regime.

For the units the condensate chemical potential $\mu^{\uparrow}=$ $n_{0} U^{\uparrow \uparrow}$, the corresponding healing length $\xi$, and the boson mass $m_{\uparrow}$ are used. In these units the self-energy exhibits a $\left(n_{0} \xi^{3}\right)^{-1}$ dependence on the density, and in the figures below the self-energy is always multiplied with this factor, which for the considered system is $n_{0} \xi^{3} \approx 14.5$.

In Fig. 2 the dispersions of the mean-field quasiparticles (17) are presented as a function of the applied magnetic field $B$ at $k=0$. Note that due to the presence of the condensate
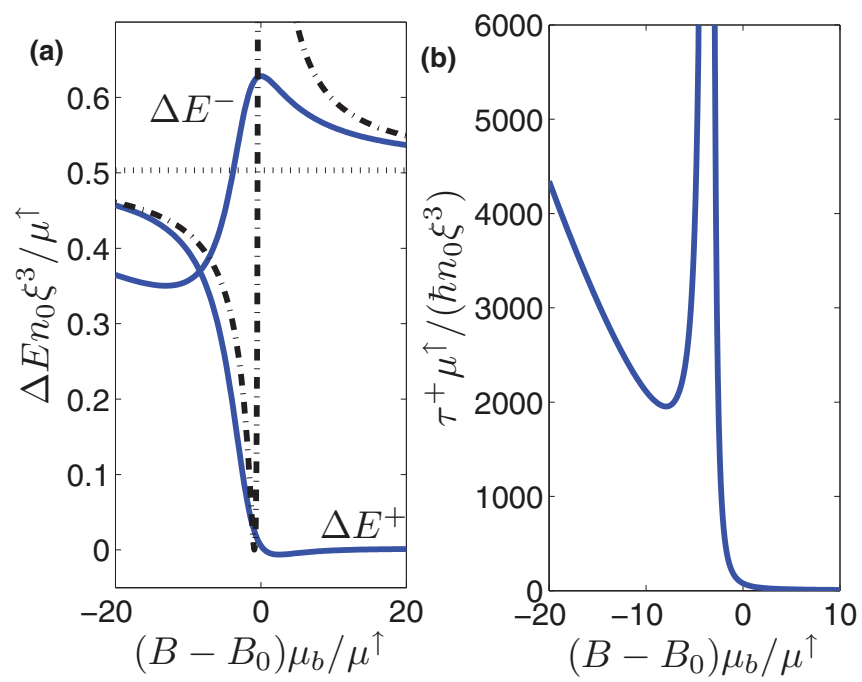

FIG. 3. (Color online) (a) The perturbative correction $\Delta E^{ \pm}$to the mean-field quasiparticle energies from Fig. 2 as a function of the magnetic field $B$ at $k=0$ for a ${ }^{6} \mathrm{Li}$ impurity in a Na condensate. The dotted line is the background result (45) and the dot-dashed line is the perturbative single-channel result. (b) The corresponding lifetime of the upper branch.

the resonance is shifted from $B_{0}$ to

$$
B_{0}^{*}=B_{0}+\frac{n_{0}}{\delta \mu}\left(\frac{2 \pi \hbar^{2} a_{\mathrm{BG}}}{m_{r}}+\frac{4 \pi \hbar^{2} a_{\mathrm{BB}}}{m_{\uparrow}}\right),
$$

which for the system under consideration gives $\left(B_{0}^{*}-B_{0}\right) \mu_{b} /$ $\mu^{\uparrow} \approx-2.21$. Also the result of the perturbative single-channel calculation is presented, which strongly deviates from the perturbative result around the resonance and diverges at $B=$ $B_{0}$. The single-channel result provides a good approximation far from the resonance for the branch with energy closest to the impurity energy (which we will denote as the impuritylike branch). This could be expected since in this case the occupation of the closed channel is small.

In Fig. 3(a) the perturbative corrections $\Delta E^{ \pm}=$ $\operatorname{Re}\left[\hbar \Sigma^{ \pm}\left(0, \varepsilon_{\vec{k}}^{ \pm}\right)\right]$to the mean-field energies, as defined in Eq. (28), are presented as a function of the magnetic field $B$ at $k=0$. The background result (45) and the perturbative single-channel result are also shown. Again the perturbative single-channel result diverges at $B=B_{0}$ and is only a good approximation for the impuritylike branch far from the resonance. In Fig. 3(b) the corresponding lifetime (29) of the upper branch is presented. The lifetime is infinite in the limit $B \rightarrow-\infty$ (impuritylike) and zero for $B \rightarrow \infty$ (molecularlike). In the vicinity of the resonance a strong peak is observed with a maximum corresponding to an infinite lifetime at $\left(B-B_{0}\right) \mu_{b} / \mu^{\uparrow} \approx-3.55$.

The lifetimes of the two branches are presented in Fig. 4 as a function of the wave number $k$ at $B=B_{0}$. Also the lifetime in the absence of a molecule is presented [the inverse of Eq. (46)] which is only finite for $k>k_{c}$, with $k_{c}$ the Landau critical value. For the lower branch a similar behavior is observed with an infinite lifetime at small $k$ until the Landau critical value $k_{c}^{-}$is reached. This value is the lowest possible value of $k$ that satisfies conservation of energy and momentum for the 

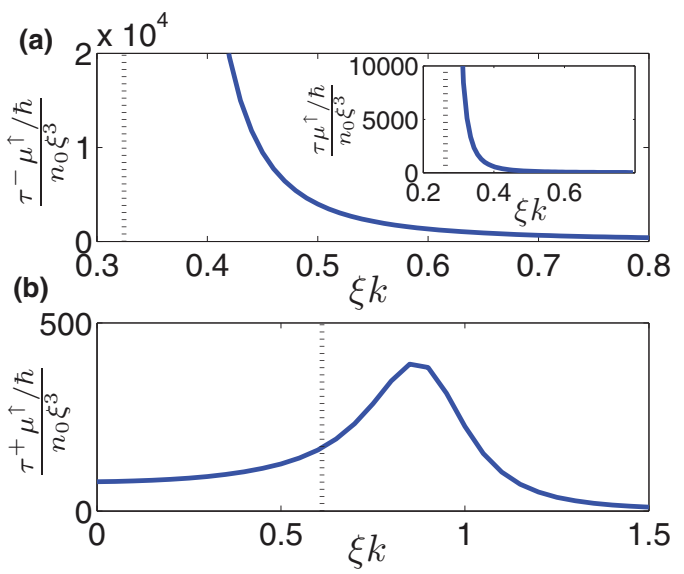

FIG. 4. (Color online) The lifetimes of the lower (a) and the upper (b) quasiparticle branches are presented as a function of the wave number $k$ for a ${ }^{6} \mathrm{Li}$ impurity in a $\mathrm{Na}$ condensate. The inset in (a) shows the lifetime in the absence of a molecule. The dotted lines indicate the landau critical values.

emission of a Bogoliubov excitation and is thus determined by

$$
k_{c}^{ \pm}=\min \left\{k=|\vec{k}|: \exists \vec{q} \mid \varepsilon_{\vec{k}}^{ \pm}=\varepsilon_{\vec{k}-\vec{q}}^{ \pm}+\varepsilon_{\vec{q}}^{\mathrm{Bog}}\right\} .
$$

For the upper branch a finite lifetime is also found at small $k$ since it can also decay to the lower branch with the emission of a Bogoliubov excitation. Once $k$ reaches the Landau critical value $k_{c}^{+}$, as determined by Eq. (52), an extra decay channel opens corresponding to the emission of a Bogoliubov excitation.

In Fig. 5(a) the mean-field effective masses (18) are presented for the two quasiparticle branches, and in (b) the perturbative correction $1 / \mathrm{m}^{\text {pert }( \pm)}$ is shown [as defined in Eq. (30)]. The background result and the perturbative singlechannel result are also depicted. Again we find that the singlechannel result strongly deviates from the perturbative result in
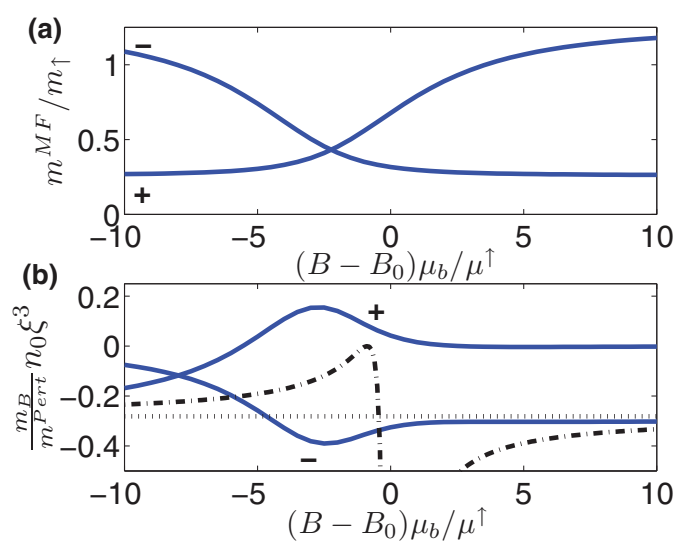

FIG. 5. (Color online) The effective masses are presented for the two branches as a function of the magnetic field $B$ for a ${ }^{6} \mathrm{Li}$ impurity in a Na condensate. In (a) the mean-field result (18) is presented and in (b) the perturbative correction to the inverse effective mass is shown. The dotted line is the background result (48) and the dash-dotted line is the perturbative singe-channel result. the vicinity of the resonance but is a reasonable approximation for the impuritylike branch far from the resonance.

\section{CONCLUSIONS}

A coupled-channel calculation is presented for the polaronic system consisting of an impurity in a Bose-condensed gas in the presence of an interspecies Feshbach resonance. At the mean-field level, two quasiparticles branches are found that consist of a mixing of the impurity and the molecular states. Due to the presence of the condensate, the location of the Feshbach resonance is shifted. The corrections to the mean-field result are examined within perturbation theory. This approximation is valid if both the coupling to the molecular channel and the background interactions are weak with respect to the condensate interaction energy and if the condensate healing length is sufficiently large compared to the average interparticle distance.

The model parameters are related to the experimental parameters by identifying the low-energy $T$ matrix and using a proper renormalization scheme. The results are finally discussed for the specific system of a ${ }^{6} \mathrm{Li}$ impurity atom in a Bose-condensed atomic $\mathrm{Na}$ gas in three dimensions.

Far from the resonance the results for the quasiparticle branch with an energy close to the impurity energy are well reproduced with a perturbative single-channel calculation. However, in the vicinity of the resonance the results from the two calculations strongly deviate since the perturbative single-channel calculation diverges at the resonance, while the coupled-channel calculation predicts finite results.

Some important properties of the quasiparticles, such as the lifetime and the effective mass, were examined. The lifetime of the lower branch is infinite at small wave number $k$ and becomes finite once $k$ exceeds the Landau critical value since it can then dissipate energy to the condensate. The lifetime of the upper branch is also finite at small $k$ since it can decay to the lower branch with the emission of a Bogoliubov excitation. For $k$ larger than the Landau critical value it can also emit a Bogoliubov excitation which results in two contributions to the (inverse) lifetime.

\section{APPENDIX: CALCULATION OF THE SELF-ENERGY}

If the interaction representation is used the Green's function (22) can generally be written as a perturbative expansion in the interaction Hamiltonian $\widehat{H}_{I}$ as [17]

$$
\begin{aligned}
G\left(\vec{K}, t-t^{\prime}\right)= & \frac{-i}{\langle 0|U(\infty,-\infty)| 0\rangle} \sum_{n=0}^{\infty} \frac{1}{n !(i \hbar)^{n}} \\
& \times \int_{-\infty}^{\infty} d t_{1} \ldots d t_{n}\langle 0| \mathcal{T}\left[\widehat{\Psi}_{\vec{K}}(t) \widehat{H}_{I}\left(t_{1}\right) \ldots\right. \\
& \left.\times \widehat{H}_{I}\left(t_{n}\right) \widehat{\Psi}_{\vec{K}}^{\dagger}\left(t^{\prime}\right)\right]|0\rangle
\end{aligned}
$$

where $U\left(t, t_{0}\right)$ denotes the time evolution operator:

$$
U\left(t, t_{0}\right)=\mathcal{T} \exp \left[\frac{1}{i \hbar} \int_{t_{0}}^{t} d t_{1} \widehat{H}_{I}\left(t_{1}\right)\right] .
$$

The effect of the denominator $\langle 0|U(\infty,-\infty)| 0\rangle$ is to cancel all contributions from disconnected diagrams from the series. 
For the considered model system with the interaction Hamiltonian (19) the first-order correction is zero since it involves the expectation value of a single creation or annihilation operator with respect to the vacuum. We are interested in the lowest nonvanishing correction, which means we have to calculate the following expectation value:

$$
\left\langle 0\left|\mathcal{T}\left[\widehat{\Psi}_{\vec{k}}(t) \widehat{H}_{I}\left(t_{1}\right) \widehat{H}_{I}\left(t_{2}\right) \widehat{\Psi}_{\vec{k}}^{\dagger}\left(t^{\prime}\right)\right]\right| 0\right\rangle .
$$

\section{Expectation values of the quasiparticle operators}

All the needed expectation values are of the following form:

$$
\begin{aligned}
& \langle 0| \mathcal{T}\left[\widehat{\Psi}_{\vec{K}}(t)\left(\gamma_{\vec{k}_{1}+\vec{q}_{1}} \widehat{\Phi}_{\vec{k}_{1}+\vec{q}_{1}}^{\dagger}\left(t_{1}\right)+\sigma_{\vec{k}_{1}+\vec{q}_{1}} \widehat{\Psi}_{\vec{k}_{1}+\vec{q}_{1}}^{\dagger}\left(t_{1}\right)\right)\left(\beta_{k_{1}} \widehat{\Phi}_{\vec{k}_{1}}\left(t_{1}\right)-\alpha_{k_{1}} \widehat{\Psi}_{\vec{k}_{1}}\left(t_{1}\right)\right)\right. \\
& \left.\quad \times\left(\gamma_{\vec{k}_{2}+\vec{q}_{2}} \widehat{\Phi}_{\vec{k}_{2}+\vec{q}_{2}}^{\dagger}\left(t_{2}\right)+\sigma_{\vec{k}_{2}+\vec{q}_{2}} \widehat{\Psi}_{\vec{k}_{2}+\vec{q}_{2}}^{\dagger}\left(t_{2}\right)\right)\left(\beta_{k_{2}} \widehat{\Phi}_{\vec{k}_{2}}\left(t_{2}\right)-\alpha_{k_{2}} \widehat{\Psi}_{\vec{k}_{2}}\left(t_{2}\right)\right) \widehat{\Psi}_{\vec{K}}^{\dagger}\left(t^{\prime}\right)\right]|0\rangle .
\end{aligned}
$$

First the contribution with six creation or annihilation operators for the lower branch is considered:

$$
\sigma_{\vec{k}_{1}+\vec{q}_{1}} \alpha_{k_{1}} \sigma_{\vec{k}_{2}+\vec{q}_{2}} \alpha_{k_{2}}\left\langle 0\left|\mathcal{T}\left[\widehat{\Psi}_{\vec{K}}(t) \widehat{\Psi}_{\vec{k}_{1}+\vec{q}_{1}}^{\dagger}\left(t_{1}\right) \widehat{\Psi}_{\vec{k}_{1}}\left(t_{1}\right) \widehat{\Psi}_{\vec{k}_{2}+\vec{q}_{2}}^{\dagger}\left(t_{2}\right) \widehat{\Psi}_{\vec{k}_{2}}\left(t_{2}\right) \widehat{\Psi}_{\vec{K}}^{\dagger}\left(t^{\prime}\right)\right]\right| 0\right\rangle
$$

This can be rewritten with Wick's theorem. We will only consider connected diagrams since the disconnected cancel with the denominator in Eq. (A1), and because we are interested in a single particle at zero temperature the expectation value of the occupation number is taken as zero. This results in only two contributions:

$$
\begin{aligned}
& \sigma_{\vec{k}_{1}+\vec{q}_{1}} \alpha_{k_{1}} \sigma_{\vec{k}_{2}+\vec{q}_{2}} \alpha_{k_{2}}\left\langle 0\left|\mathcal{T}\left[\widehat{\Psi}_{\vec{K}}(t) \widehat{\Psi}_{\vec{k}_{1}+\vec{q}_{1}}^{\dagger}\left(t_{1}\right) \widehat{\Psi}_{\vec{k}_{1}}\left(t_{1}\right) \widehat{\Psi}_{\vec{k}_{2}+\vec{q}_{2}}^{\dagger}\left(t_{2}\right) \widehat{\Psi}_{\vec{k}_{2}}\left(t_{2}\right) \widehat{\Psi}_{\vec{K}}^{\dagger}\left(t^{\prime}\right)\right]\right| 0\right\rangle \\
& \quad \rightarrow \sigma_{\vec{K}} \alpha_{\vec{K}} \alpha_{k_{1}} \sigma_{\vec{k}_{1}} i G_{0}^{-}\left(\vec{K}, t-t_{1}\right) i G_{0}^{-}\left(\vec{k}_{1}, t_{1}-t_{2}\right) i G_{0}^{-}\left(\vec{K}, t_{2}-t^{\prime}\right) \delta_{\vec{K}, \vec{k}_{1}+\vec{q}_{1}} \delta_{\vec{k}_{1}, \vec{k}_{2}+\vec{q}_{2}} \delta_{\vec{k}_{2}, \vec{K}} \\
& \quad+\alpha_{\vec{K}} \sigma_{\vec{K}} \sigma_{\vec{k}_{2}} \alpha_{k_{2}} i G_{0}^{-}\left(\vec{K}, t-t_{2}\right) i G_{0}^{-}\left(\vec{k}_{2}, t_{2}-t_{1}\right) i G_{0}^{-}\left(\vec{K}, t_{1}-t^{\prime}\right) \delta_{\vec{K}, \vec{k}_{2}+\vec{q}_{2}} \delta_{\vec{k}_{2}, \vec{k}_{1}+\vec{q}_{1}} \delta_{\vec{k}_{1}, \vec{K}},
\end{aligned}
$$

with $G_{0}^{ \pm}(\vec{q}, \omega)$ the Green's function of the unperturbed quasiparticles, which in the frequency representation is given by

$$
G_{0}^{ \pm}(\vec{q}, \omega)=\frac{\hbar}{\hbar \omega-\varepsilon_{\vec{k}}^{ \pm}+i \delta},
$$

where $\varepsilon_{\vec{k}}^{ \pm}$are the mean-field quasiparticle dispersions (17). There are four terms with four creation or annihilation operators for the lower branch for which Wick's theorem can be used again. Again we only consider the connected diagrams for a single particle at zero temperature, which results in the following two terms:

$$
\begin{aligned}
& \sigma_{\vec{K}} \beta_{k_{1}} \gamma_{\vec{k}_{1}} \alpha_{\vec{K}} i G_{0}^{-}\left(\vec{K}, t-t_{1}\right) i G_{0}^{-}\left(\vec{K}, t_{2}-t^{\prime}\right) i G_{0}^{+}\left(\vec{k}_{1}, t_{1}-t_{2}\right) \delta_{\vec{K}, \vec{k}_{1}+\vec{q}_{1}} \delta_{\vec{k}_{2}, \vec{K}} \delta_{\vec{k}_{1}, \vec{k}_{2}+\vec{q}_{2}} \\
& \quad+\gamma_{\vec{k}_{2}} \alpha_{\vec{K}} \sigma_{\vec{K}} \beta_{k_{2}} i G_{0}^{-}\left(\vec{K}, t-t_{2}\right) i G_{0}^{-}\left(\vec{K}, t_{1}-t^{\prime}\right) i G_{0}^{+}\left(\vec{k}_{2}, t_{2}-t_{1}\right) \delta_{\vec{K}, \vec{k}_{2}+\vec{q}_{2}} \delta_{\vec{k}_{1}, \vec{K}} \delta_{\vec{k}_{1}+\vec{q}_{1}, \vec{k}_{2}} .
\end{aligned}
$$

Note that the term with only two creation and annihilation operators of the lower branch represents a disconnected diagram and thus also cancels with the denominator of Eq. (A1), which means we have all the different contributions for the expectation value (A4).

\section{Expectation values of the Bogoliubov operators}

Typically expectation values of the following form are needed:

$$
\begin{aligned}
& \left\langle 0\left|\mathcal{T}\left[\left(u_{\vec{q}_{1}} \widehat{\alpha}_{\vec{q}_{1}}\left(t_{1}\right)-v_{\vec{q}_{1}} \widehat{\alpha}_{-\vec{q}_{1}}^{\dagger}\left(t_{1}\right)\right)\left(u_{\vec{q}_{2}} \widehat{\alpha}_{\vec{q}_{2}}\left(t_{2}\right)-v_{\vec{q}_{2}} \widehat{\alpha}_{-\vec{q}_{2}}^{\dagger}\left(t_{2}\right)\right)\right]\right| 0\right\rangle \\
& \quad=-u_{\vec{q}_{1}} v_{\vec{q}_{1}}\left[i D\left(\vec{q}_{1}, t_{1}-t_{2}\right)+i D\left(-\vec{q}_{1}, t_{2}-t_{1}\right)\right] \delta_{\vec{q}_{1},-\vec{q}_{2}} .
\end{aligned}
$$

Here $D\left(\vec{q}, t_{1}-t_{2}\right)$ denotes the unperturbed Green's function for the Bogoliubov excitations. In frequency representation it is given by

$$
D(\vec{q}, \omega)=\frac{\hbar}{\hbar \omega-\varepsilon_{\vec{k}}^{\text {Bog }}+i \delta},
$$

with $\varepsilon_{\vec{k}}^{\text {Bog }}$ the Bogoliubov dispersion (9). 


\section{Expression for the self-energy}

Bringing everything together gives the following result:

$$
\begin{aligned}
\int_{-\infty}^{\infty} & d t_{1} \int_{-\infty}^{\infty} d t_{2}\left\langle 0\left|\mathcal{T}\left[\widehat{\Psi}_{\vec{K}}(t) \widehat{H}_{I}\left(t_{1}\right) \widehat{H}_{I}\left(t_{2}\right) \widehat{\Psi}_{\vec{K}}^{\dagger}\left(t^{\prime}\right)\right]\right| 0\right\rangle \\
= & 2 \int_{-\infty}^{\infty} d t_{1} \int_{-\infty}^{\infty} d t_{2} \sum_{\vec{q}} G_{0}^{-}\left(\vec{K}, t-t_{1}\right) G_{0}^{-}\left(\vec{K}, t_{2}-t^{\prime}\right) \\
& \times\left\{\left[\left(u_{\vec{q}} \sigma_{\vec{K}} \alpha_{\vec{K}-\vec{q}}-v_{\vec{q}} \alpha_{\vec{K}} \sigma_{\vec{K}-\vec{q}}\right)^{2} D\left(\vec{q}, t_{1}-t_{2}\right)\right.\right. \\
& \left.+\left(v_{\vec{q}} \sigma_{\vec{K}} \alpha_{\vec{K}-\vec{q}}-u_{\vec{q}} \alpha_{\vec{K}} \sigma_{\vec{K}-\vec{q}}\right)^{2} D\left(-\vec{q}, t_{2}-t_{1}\right)\right] G_{0}^{-}\left(\vec{K}-\vec{q}, t_{1}-t_{2}\right) \\
& +\left[\left(u_{\vec{q}} \sigma_{\vec{K}} \beta_{\vec{K}-\vec{q}}+v_{\vec{q}} \gamma_{\vec{K}-\vec{q}} \alpha_{\vec{K}}\right)^{2} D\left(\vec{q}, t_{1}-t_{2}\right)\right. \\
& \left.\left.+\left(v_{\vec{q}} \sigma_{\vec{K}} \beta_{\vec{K}-\vec{q}}+u_{\vec{q}} \gamma_{\vec{K}-\vec{q}} \alpha_{\vec{K}}\right)^{2} D\left(-\vec{q}, t_{2}-t_{1}\right)\right] G_{0}^{+}\left(\vec{K}-\vec{q}, t_{1}-t_{2}\right)\right\}
\end{aligned}
$$

By going to the frequency representation with a Fourier transformation with respect to the time, as in Eq. (23), the following expression is retrieved for the second-order correction to the Green's function of the lower quasiparticle branch:

$$
G^{-(2)}(\vec{k}, \omega)=G_{0}^{-}(\vec{K}, \omega) \Sigma^{-(2)}(\vec{k}, \omega) G_{0}^{-}(\vec{K}, \omega),
$$

with the self-energy $\Sigma^{-(2)}(\vec{k}, \omega)$ given by

$$
\begin{aligned}
\Sigma^{-(2)}(\vec{k}, \omega)= & \frac{i}{\hbar^{2}} \int_{-\infty}^{\infty} \frac{d v}{2 \pi} \sum_{\vec{q}}\left\{\left[\left(u_{\vec{q}} \sigma_{\vec{K}} \alpha_{\vec{K}-\vec{q}}-v_{\vec{q}} \alpha_{\vec{K}} \sigma_{\vec{K}-\vec{q}}\right)^{2} D(\vec{q}, v)\right.\right. \\
& \left.+\left(v_{\vec{q}} \sigma_{\vec{K}} \alpha_{\vec{K}-\vec{q}}-u_{\vec{q}} \alpha_{\vec{K}} \sigma_{\vec{K}-\vec{q}}\right)^{2} D(-\vec{q},-v)\right] G_{0}^{-}(\vec{K}-\vec{q}, \omega-v) \\
& +\left[\left(u_{\vec{q}} \sigma_{\vec{K}} \beta_{\vec{K}-\vec{q}}+v_{\vec{q}} \gamma_{\vec{K}-\vec{q}} \alpha_{\vec{K}}\right)^{2} D(\vec{q}, v)\right. \\
& \left.\left.+\left(v_{\vec{q}} \sigma_{\vec{K}} \beta_{\vec{K}-\vec{q}}+u_{\vec{q}} \gamma_{\vec{K}-\vec{q}} \alpha_{\vec{K}}\right)^{2} D(-\vec{q},-v)\right] G_{0}^{+}(\vec{K}-\vec{q}, \omega-v)\right\} .
\end{aligned}
$$

Using the Dyson equation it is clear that the self-energy $\Sigma(\vec{k}, \omega)$ from Eq. (24) can be approximated by the self-energy (A13). The frequency integral in Eq. (A13) can be done with contour integration, which finally results in expression (25a) for the self-energy of the lower branch. For the upper branch a similar calculation can be done, resulting in expression (25b).

[1] I. Bloch, J. Dalibard, and W. Zwerger, Rev. Mod. Phys. 80, 885 (2008).

[2] C. Chin, R. Grimm, P. Julienne, and E. Tiesinga, Rev. Mod. Phys. 82, 1225 (2010).

[3] R. Feynman, Int. J. Theor. Phys. 21, 467 (1982).

[4] F. M. Cucchietti and E. Timmermans, Phys. Rev. Lett. 96, 210401 (2006).

[5] R. M. Kalas and D. Blume, Phys. Rev. A 73, 043608 (2006).

[6] J. Tempere, W. Casteels, M. K. Oberthaler, S. Knoop, E. Timmermans, and J. T. Devreese, Phys. Rev. B 80, 184504 (2009).

[7] N. Spethmann, F. Kindermann, S. John, C. Weber, D. Meschede, and A. Widera, Phys. Rev. Lett. 109, 235301 (2012).

[8] T. Takekoshi, M. Debatin, R. Rameshan, F. Ferlaino, R. Grimm, H.-C. Nägerl, C. R. Le Sueur, J. M. Hutson, P. S. Julienne, S. Kotochigova, and E. Tiemann, Phys. Rev. A 85, 032506 (2012).

[9] T. Schuster, R. Scelle, A. Trautmann, S. Knoop, M. K. Oberthaler, M. M. Haverhals, M. R. Goosen, S. J. J. M. F. Kokkelmans, and E. Tiemann, Phys. Rev. A 85, 042721 (2012).

[10] I. Carusotto, T. Volz, and A. Imamoglu, Europhys. Lett. 90, 37001 (2010).
[11] N. Takemura, S. Trebaol, M. Wouters, M. T. Portella-Oberli, and B. Deveaud, Nat. Phys. 10, 500 (2014).

[12] M. Wouters, Phys. Rev. B 76, 045319 (2007).

[13] R. A. Duine and H. T. C. Stoof, Phys. Rep. 396, 115 (2004).

[14] S. J. J. M. F. Kokkelmans, J. N. Milstein, M. L. Chiofalo, R. Walser, and M. J. Holland, Phys. Rev. A 65, 053617 (2002).

[15] E. Timmermans, P. Tommasini, M. Hussein, and A. Kerman, Phys. Rep. 315, 199 (1999).

[16] L. Pitaevskii and S. Stringari, Bose-Einstein Condensation (Clarendon, Oxford, 2003).

[17] G. D. Mahan, Many-Particle Physics, 2nd ed. (Plenum, New York, 1993).

[18] H. Bei-Bing and W. Shao-Long, Chin. Phys. Lett. 26, 080302 (2009).

[19] A. Novikov and M. Ovchinnikov, J. Phys. A 42, 135301 (2009).

[20] L. Landau, Phys. Rev. 60, 356 (1941).

[21] S. Knoop, T. Schuster, R. Scelle, A. Trautmann, J. Appmeier, M. K. Oberthaler, E. Tiesinga, and E. Tiemann, Phys. Rev. A 83, 042704 (2011). 